\title{
LOW COST LOW POWER ELECTROOCULOGRAM BASED HUMAN MACHINE INTERFACE
}

\author{
Saravanan A ${ }^{1}$, Silpa Ajith Kumar ${ }^{2}$ \\ ${ }^{1}$ Student, MTech, Microelectronics and Control Systems, DayanandaSagar College of Engineering, Bangalore, India \\ Sarvy92@gmail.com \\ ${ }^{2}$ Associate Professor, Department of Electronics and Instrumentation Engineering, Dayananda Sagar College of \\ Engineering, Bangalore, India \\ silpaajithkumar@gmail.com
}

\begin{abstract}
The development of assistive technologies by means of human machine interface is turning out to be one of the most attractive areas of today's research. Innovation in power consumption, signal processing and wireless communication has been the focus for the development of such systems. However, these devices have not reached the mass market yet. The development of a versatile system which can as well play a major role in clinical and rehabilitative control applications could revolutionize this domain. The system is based on TI's embedded processor, wireless communication solutions and highly-customized analog front ends. As a proof of concept, our technology integrates the MSP430 architecture, INA333-HT instrumentation amplifier, INA129/128 as analog front end and further LM324 for analog signal processing in an effective manner. This system measures EOG, a biopotential signal, which is being pre-processed, amplified, digitized and after signal processing results are wirelessly transmitted thereby establishing human-machine communication. This project cleverly combines hardware and software disciplines resulting into one successful system which could hold ground for the development of intelligent assistive technology. The proposed technology holds clinical and rehabilitative applications as well.
\end{abstract}

Keywords: EOG; Paralysis; Epithelium; HMI; Assistive Technology; Bio-Potential $* * *$

\section{INTRODUCTION}

Physical impairments come in many forms and can generally be classified as a loss or limitation of function in muscle control or movement or a limitation in mobility. This may include hands that are too large or small for a keyboard, shakiness, arthritis, paralysis, and limb loss, among other difficulties. There is a wide range of assistive technologies available to help with all these impairments. One among the several such technologies is the human machine interface by means of EOG signals. The eye movements have attracted many researchers in the past. However since the electrooculography (EOG) provides an effective, low cost and noninvasive method for detection of full range of eye movements it has been a frequently used system in ophthalmoscopy. It is as well as used to assess the function of pigment epithelium. Besides the clinical applications of EOG, it has been the theme of research for development of assistive technologies by human-machine interface $[1,2]$.

The electro-oculogram is the measure of potential between the cornea and the retina of the eye called the corneal-retinal potential (CRP). This potential is generated due to the hyper-polarization and depolarization of retinal cells. During the process the cornea establishes a relatively positive potential with respect to the retina. The electrodes are placed round the eyes to acquire the changing position. The EOG is acquired using $\mathrm{Ag}-\mathrm{AgCl}$ electrode which is placed as a single channel electrode placement system namely the horizontal channel. The strength of the EOG signal varies from $10-30 \mathrm{mV}$ [3] with frequencies in the $\mathrm{DC}-10 \mathrm{~Hz}$ band [4]. In addition to EOG signal produced from the eyeball rotation and movements it is also affected by different sources of artifacts like head movements, electroencephalogram (EEG) signals, electrode placement thereby resulting in noise. Hence, it becomes necessary to develop an amplifier which eliminates the shifting resting potentials arising due to these artifacts and simultaneously avoid saturation. Most of the commercially available EOG amplifiers are costly and uses intricate design. Hence, the development of dedicated topology for the maximization of overall circuit performance in a cost effective manner has been the motive behind this study. Additionally, this system follows the trend of integrating real-time embedded systems with wireless transmission for diagnostics [5, 6], making it more practicable to implement on newer and smaller platforms.

Note: This is not based on eye blink sensor which uses photodiode and LED. It is based on EOG and signal processing.

\section{EXISITING SYSTEM}

Recent technological research which is being carried out in this field is on the "Design of wireless EOG Bio-potential amplifier". The focus is on designing a low cost amplifier to amplify the signal obtained as a result of movement of eye. Although human machine interface has been established by means of image processing, camera interface techniques 
using MATLAB or LabView it has been found that the overall cost factor and power consumption is more in laptop or tablet to perform this action. Hence this drawback of cost factor, power consumption level and mobility is been effectively targeted by the proposed system.

By eliminating the usage of MATLAB and laptop we are able to cut down the overall unit cost of the system by $1 / 20^{\text {th }}$ of the original market price.

\section{PROPOSED SYSTEM}

This system measures EOG, a bio-potential signal, by means of electrodes which is placed as shown in figure 1 and is being pre-processed, amplified, digitized and after signal processing results are wirelessly transmitted thereby establishing human-machine communication. Along with this module an integrated power supply unit has also been effectively designed to establish a very low power consumption device. This project cleverly combines hardware and software disciplines resulting into one successful system which could hold ground for the development of intelligent assistive technology [7, 8].

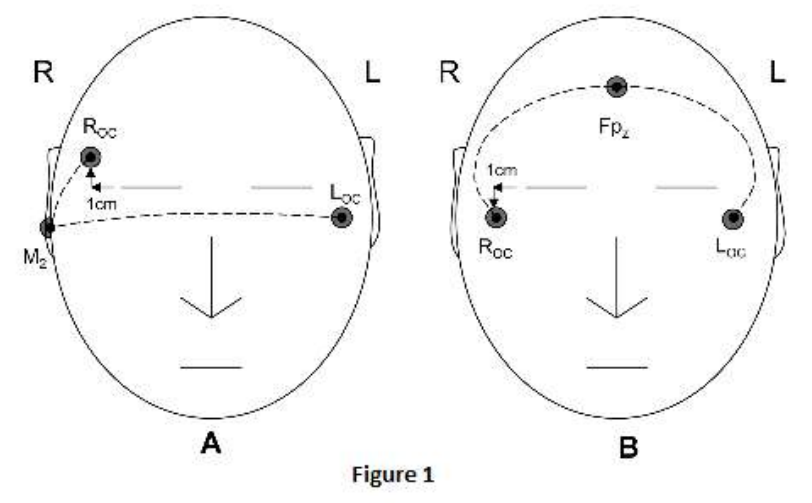

\section{IMPLEMENTATION}

The overall workflow is as follows which is depicted through the following Block Diagram (Figure 2).

Figure 2 Eye movement detector and transmitter
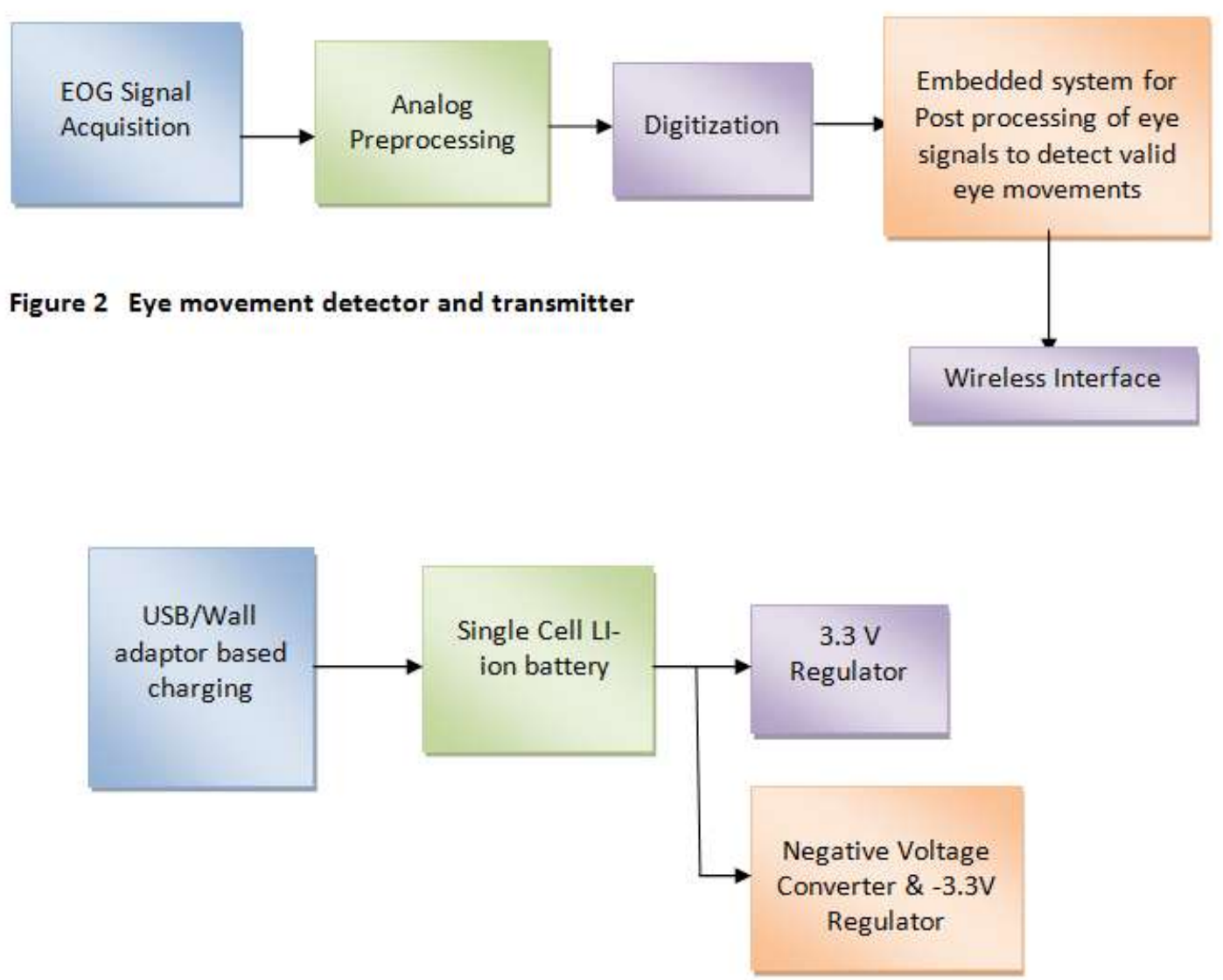

Figure 3 Power System for EOG based eye movement detection system 


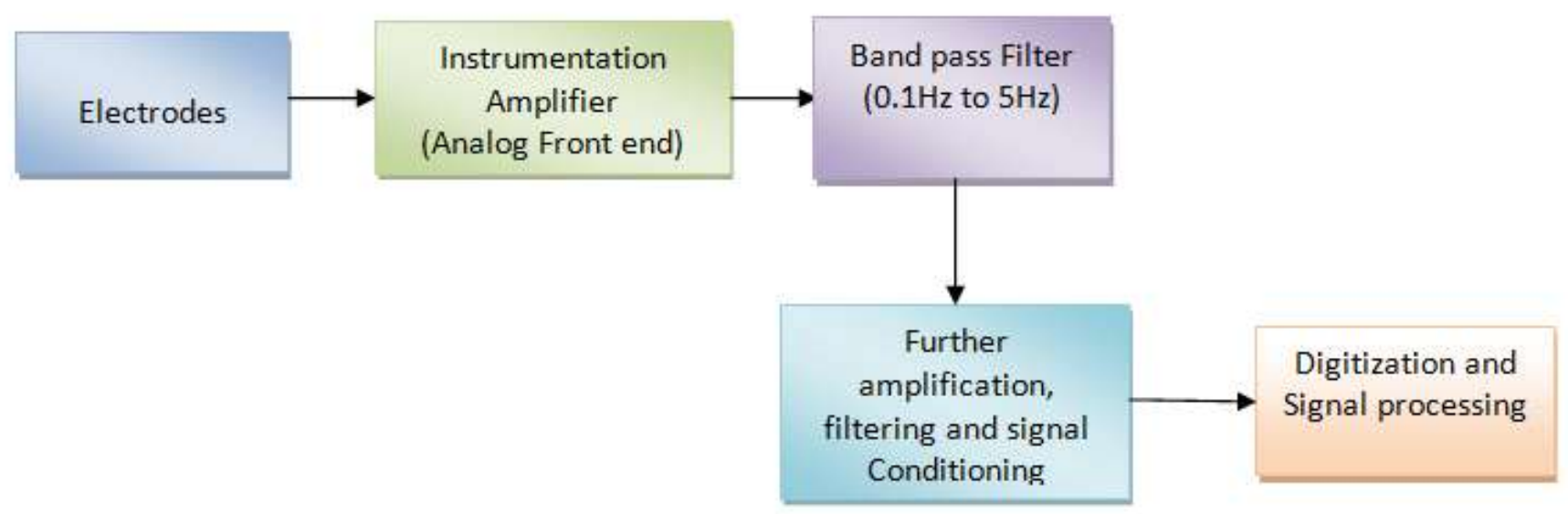

Figure 4 EOG Acquisition system for eye movement detection

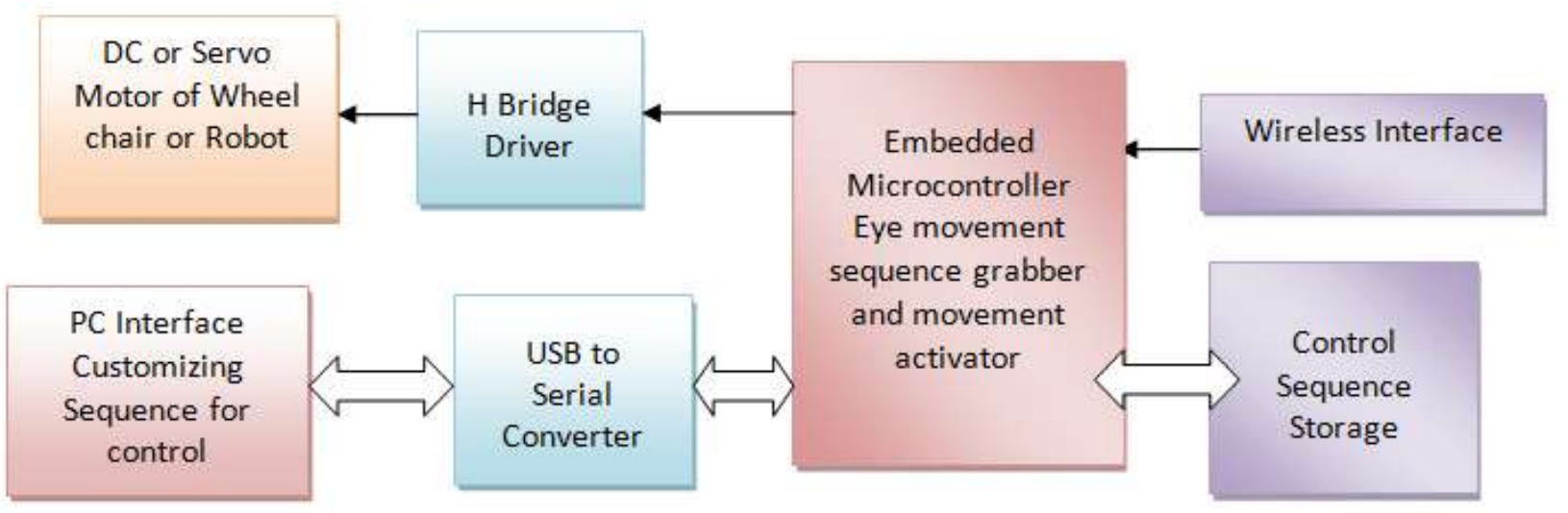

Figure 5 Eye movement Sequence identifier and motion control system

Description: This project is divided into 4 parts: power system design, Robot design, Command receiver and eye movement detection and transmission (Figure 3 and Figure 4). Robot is used in place of wheel chair since wheel chair price starts from 50 thousand rupees. Robot is built using two DC servo motor which is controlled by $\mathrm{H}$ bridge current driver. Microcontroller based upon the command received from transmission part, controls the direction of the motors and powers the motors to achieve left, right, forward and reverse movements (Figure 5).

Command receiver is basically a sequential decoder which grabs the eye movement received from the TX part using wireless interface. The command sequence to robot movement conversion is customizable and it can be customized with the help of PC by means of serial interface and hence if $\mathrm{PC}$ is not having serial interface a USB to serial converter is required to customize the command. The command sequence for each robot/wheelchair movement is stored in EEPROM and this memory acts as control sequence storage. This is interfaced and accessed using I2C protocol and if EEPROM is internal to controller then external EEPROM is not required.

Like various other technologies Bio interface technology is taking shape and finding application in various aspect of life. One such technology is the eye movement detection thereby we are using this proven technology to get eye movement after processing EOG signals in analog circuit and digital signal processing. These eye movements are transmitted using wireless interface to command receiver.

\section{RESULTS}

The final analog circuit design (Figure 6) is as shown below which produces a DC shifted signal as the output and enables to control the robot wirelessly using CC2500 transceiver module. 


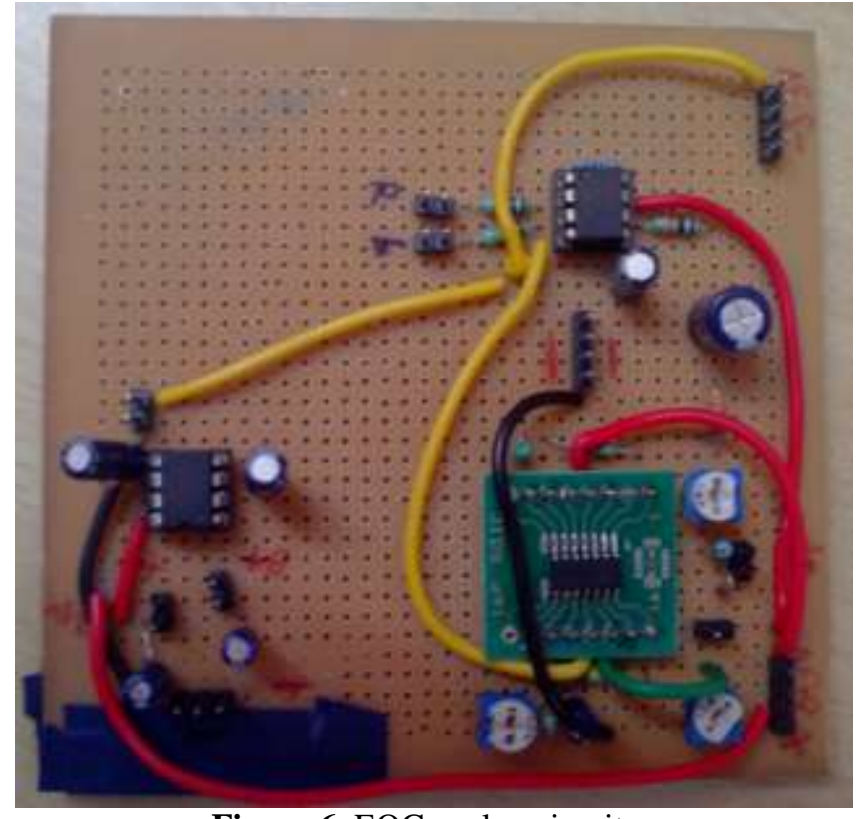

Figure 6. EOG analog circuitry

\section{CONCLUSION}

With the proposed system EOG signal is been acquired and by means of which the robot is been controlled thereby implementing with minimized calibration and detection errors. With the help of TI's MSP430 architecture, instrumentation amplifiers and a well-designed optimized power conditioning circuit the overall power consumption of the system has been reduced to great extent. Also as a part of our project the human robot interface has also been developed wherein the locomotive part of robot is being driven by means of vital signals generated by human eye. Considering this proposed system as a basis our platform can quickly be adapted to multifarious cutting-edge, seamlessly integrated, health accessories in the future. As a whole, great prospects lie ahead for the current project which can serve as a base for controlling wireless motorized wheelchair that could turn out to be a good assistive technique for people suffering from extremely limited peripheral mobility.

\section{REFERENCES}

[1] R. Barea, L. Boquete, J.M. Rodríguez-Ascariz, S. Ortega, and E. Lopez, "Sensory System for Implementing a Human-Computer Interface Based on Electrooculography," Sensors, vol. 11(1), pp. 310-328, Dec 2010

[2] M. Hashimoto, K. Takahashi, and M. Shimada, "Wheelchair control using an EOG- and EMG-based gesture interface", IEEE Proc. Advanced Intelligent Mechatronics, pp. 1212-1217, Sep 2009.

[3] T. Gandhi, M. Trikha, J. Santhosh, and S. Anand, "Development of an expert multitask gadget controlled by voluntary eye movements," Expert Syst. with Applications, vol. 37, pp. 4204-4211, 2010.

[4] S. R. Choudhury, S. Venkataramanan, H. B. Nemade, and J. S. Sahambi, "Design and Development of a
Novel EOG Biopotential Amplifier," J Bioelectromagnatism, 2004.

[5] Y. H. Nam, Z. Halm, Y. J. Chee, and K. S. Park, "Development of sensing diagnosis system integrating digital telemetry for medicine," Proc. 20th Annu. Znt. Conf. IEEE Eng. Med. Biol.Soc., vol. 20, pp. 11701173, 1998.

[6] S. Kondra, C. Yew, F. Ahmed, and U. G. Hofmann, "Prototype of a patient monitoring device based on an embedded RISC/DSP system," Proc. 39th Ann. Cong, German Society Biomed. Eng, Nuremberg, Sep 2005.

[7] http://www.enotes.com/research-starters/assistivetechnology-education

[8] G. Gargiulo, P. Bifulco, R. Calvo, M. Cesarelli, C. Jin, and A. v. Schaik, "Mobile biomedical sensing with dry electrodes," in Proc. Int. Conf. Intelligent Sensors, Sensor Networks Information Processing, ISSNIP, 2008, pp. 261-266 